\title{
Mammographic Findings in Different Breast Cancer Subtypes (luminal - Her2 positive - Triple Negative)
}

\author{
Leila Pourali ${ }^{1}$, Ali Taghizadeh ${ }^{2}$, Soudabeh Shahid Sales ${ }^{3,}$ \\ ${ }^{1}$ Department of Obstetrics and Gynecology, Assistant professor, Department of \\ Obstetrics and Gynecology, Women's Health Research Center, Ghaem Hospital, \\ Mashhad University of Medical Sciences, Mashhad, Iran \\ ${ }^{2}$ Department of Clinical Oncology, Associate professor, Department of Clinical \\ Oncology, Surgical Oncology Research Center, Imam Reza Hospital, Mashhad \\ University of Medical Sciences, Mashhad, Iran \\ ${ }^{3}$ Department of Clinical Oncology-Assistant professor, Department of Clinical \\ Oncology, Cancer Research Center, Mashhad University of Medical Sciences, \\ Mashhad, Iran \\ ${ }^{*}$ Corresponding author: Soudabeh Shahid Sales, Department of Clinical Oncology- \\ Assistant professor, Department of Clinical Oncology, Cancer Research Center, \\ Mashhad University of Medical Sciences, Mashhad, Iran.E-mail: shahidsaless@ \\ mums.ac.ir
}

DOI: $10.21859 / \mathrm{mci}$-supp-116

\section{Keywords:}

Breast Cancer

Immunohistochemistry

Mammography

\begin{abstract}
Introduction: Prognosis and management of breast cancer are defined by different variables including histological type, grading, clinical stage, Her2+, estrogen and progesterone receptor conditions. Generally, mammography is one of the most important imaging which is done in breast cancer patients. The aim of this research was to evaluate different mammographic patterns in different breast cancer sub types.

Materials and Methods: Demographic and clinicopathologic data of 128 breast cancer patients which referred to two academic hospitals were obtained from their registered files and their mammographies were reviewed by two radiologists separately. Patients were categorized into 3 groups of Luminal, triple negative and Her2+ and the checklists were filled out by research team. The mentioned data was then analyzed by the SPSS software version 16.

Results: In this study we found significant difference in margins' clarity and axillary lymphadenopathy between mammographic presentations of different breast cancer subtypes; but the difference of other mamographic presentations including mass existence, mass size, margin type, peleomorphic calcification, micro calcification, nipple retraction, skin thickening and tissue distortion were not significantly different between three groups. Conclusions: Results of current study showed no significant difference between mammographic features of different invasive breast cancer subtypes except for axillary lymphadenopathy and ill-defined margins. Since the majority of patients were in premenopausal status; perhaps we can say lower sensitivity of mammography in premenopausal women couldn't accurately distinguish mammographic differences between invasive breast cancer subtypes in this study.
\end{abstract}

